\title{
Utilização dos critérios de Beers para avaliação das prescrições em idosos portadores de doenças crônicas vinculados a um plano de saúde
}

\author{
Henrique Souza Barros de Oliveira*, Jamile Rafaela Poltronieri de Sousa*, \\ Ana Carolina Gariba Donis" ", Maria Elisa Gonzalez Manso"***
}

\section{Resumo}

A senescência é um processo natural do envelhecimento que altera a farmacocinética e a farmacodinâmica dos medicamentos, sendo particularmente importante quando se trata de um país de idosos como o Brasil. Associado à idade, há um aumento das doenças crônicas não transmissíveis (DCNTs) e consequentemente do uso de diversos medicamentos. A polifarmácia aumenta o risco de reações adversas, especialmente quando associado à prescrição de medicamentos potencialmente inapropriados (MPIs) para idosos. O presente estudo tem como objetivo avaliar a prescrição de MPIs por meio dos critérios de Beers de 2015 em um grupo de idosos portadores de doenças crônicas vinculados a um plano de saúde em São Paulo, Brasil. Trata-se de um estudo exploratório e descritivo, realizado mediante a análise de 5.995 prescrições médicas, de setembro a dezembro de 2015. Os medicamentos foram examinados segundo os critérios de Beers revisados em 2015. Observou-se que, das 5.995 prescrições médicas feitas a 1.428 idosos, $20,3 \%$ foram consideradas inapropriadas. Os MPIs mais prescritos foram: inibidores de bomba de prótons (IBP), 39,4\% (481); antidiabéticos, 13,3\% (162); benzodiazepínicos (BZP) de ação curta, intermediária e longa, 10,8\% (132); anti-inflamatórios não hormonais (AINEs), 10,3\% (127); e anti-histamínicos de primeira geração, 6,8\% (83). O grupo estudado apresentou um alto índice de prescrição inadequada de medicamentos em uso contínuo. Trata-se de um grupo populacional de risco, devido às suas próprias particularidades clínicas, predispondo a desfechos biopsicossociais desfavoráveis.

Palavras-chave: Assistência à saúde do idoso. Lista de medicamentos potencialmente inapropriados. Planos de saúde. Prescrições de medicamentos.

* Discente do curso de Medicina do Centro Universitário São Camilo (Cusc-SP). Diretor da Liga de Estudos do Processo do Envelhecimento (Lepe) do curso de Medicina do Cusc-SP e pesquisador visitante do CNPq/ PIBIC no Instituto Sírio-Libanês de Ensino e Pesquisa (IEP-HSL). E-mail: heeenry.barros@icloud.com

** Psicóloga. Discente do curso de Medicina do Cusc-SP. Membro da Lepe do curso de Medicina do Cusc-SP. E-mail: jami.rafa.sousa@gmail.com

***** Discente do curso de Medicina do Cusc-SP. E-mail: anacarolinagariba@hotmail.com

**** Médica. Mestre em Gerontologia, doutora em Ciências Sociais e pós-doutoranda em Gerontologia Social na Pontifícia Universidade Católica de São Paulo (PUC-SP). Pesquisadora do grupo Saúde, Cultura e Envelhecimento (CNPq/PUC-SP) e do Núcleo de Estudo e Pesquisa do Envelhecimento. Endereço para correspondência: Rua Celso de Azevedo Marques, 740/1001, Mooca, São Paulo, SP, Brasil. CEP 03122-010. E-mail: mansomeg@hotmail.com

$\rightarrow$ http://dx.doi.org/10.5335/rbceh.v14i3.7376

Recebido em: 11.09.2017. Aceito em: 09.04.2018. 


\section{Introdução}

A senescência é o processo normal de envelhecimento que promove alterações das funções orgânicas, as quais culminam com maior vulnerabilidade para quebra da homeostasia orgânica e com alterações nos processos farmacodinâmicos e farmacocinéticos dos medicamentos (CONSTANTINO; FIGUEIREDO, 2015).

No trato gastrointestinal ocorre modificação da acidez gástrica e da motilidade, alterando a absorção dos fármacos. A redução da água corporal e da produção de albumina, associada ao aumento do tecido adiposo, impacta na fase de distribuição de substâncias. O fluxo hepático também é reduzido, diminuindo a capacidade de metabolização dos medicamentos. As alterações hepáticas associadas à redução da taxa de filtração glomerular também afetam a excreção tanto de fármacos quanto de metabólitos (CONSTANTINO; FIGUEIREDO, 2015; GLASSOCK; DENIC; RULE, 2017).

No sistema cardiovascular, o miocárdio sofre progressiva degeneração de fibras musculares, reduzindo sua complacência e frequência. $\mathrm{O}$ aumento da resistência vascular periférica ocorre devido à perda da elasticidade das paredes arteriais e ao aumento do depósito de colesterol e da calcificação dos vasos, o que predispõe ao surgimento de doenças como hipertensão arterial. Além disso, o organismo torna-se menos competente na regulação da pressão arterial durante as mudanças de posição do idoso, predispondo a hipotensão ortostática (FECHINE; TROMPIERI, 2012).
O sistema musculoesquelético também sofre alterações importantes que predispõem a quedas e fraturas. Há, ainda, redução da densidade óssea e diminuição da força muscular e da massa magra, enquanto ocorre aumento da massa gorda (FECHINE; TROMPIERI, 2012).

A fisiologia da senescência torna-se ainda mais importante atualmente devido à mudança demográfica que está ocorrendo no Brasil. Um país é considerado "idoso" quando a proporção de pessoas acima de 60 anos é superior a $7 \%$ da população geral. Presentemente, esse grupo etário representa $14,3 \%$ da população geral no Brasil. Entre 2005 e 2015, os habitantes com mais de 60 anos passaram de $9,8 \%$ para $14 \%$. A perspectiva é que, em 2050, a população de idosos seja triplicada (CHAIMOWICZ et al., 2013).

A mudança demográfica trouxe um novo desafio para a medicina: lidar com a crescente prevalência de doenças crônicas não transmissíveis (DCNTs), que são enfermidades que evoluem em longo prazo e culminam em processos degenerativos que podem levar a incapacidade funcional e óbito (MANSO; GALERA, 2015).

No Brasil, a elevada prevalência de DCNTs associada tanto ao amplo acesso a medicamentos isentos de prescrição quanto à escassez de tratamentos não farmacológicos contribui para a automedicação. Associa-se a esse fator a pouca importância dada nas graduações em saúde, de maneira geral, ao estudo da senescência e da farmacoterapia em idosos, em um panorama que predispõe à utilização de medicamentos potencialmente inapropriados (MPIs) e à polifarmácia, esta 
definida como sendo a utilização contínua de cinco ou mais tipos de medicamentos. Essa prática representa um risco em potencial para o desenvolvimento de reações adversas a medicamentos (RAMs) (CARVALHO et al., 2012; GORZONI; FABBRI; PIRES, 2012).

A probabilidade de RAMs cresce quando está presente a prescrição de MPIs, ou seja, a indicação de fármacos cujos riscos de provocar efeitos colaterais são maiores do que seus benefícios (MANSO; BIFFI; GERARDI, 2015). Estima-se que 33\% dos idosos no Brasil apresentem RAMs, por isso, torna-se imprescindível identificar e estudar essas medicações. Para esse fim, foram desenvolvidos instrumentos que avaliam a terapia farmacológica em idosos. No entanto, no Brasil, esses instrumentos ainda são pouco conhecidos por médicos não geriatras (AMERICAN GERIATRICS SOCIETY, 2012).

Um desses instrumentos é o critério de Beers, criado em 1991, que compilou uma lista de medicamentos considerados inapropriados para utilização em idosos, sendo sua versão mais atual a publicada em 2015 (MANSO; BIFFI; GERARDI, 2015).

Pesquisas revelam que a prescrição de MPIs, assim classificados segundo o critério de Beers, está relacionada a desfechos negativos, tais como delirium, sangramentos gastrointestinais, quedas e fraturas (AMERICAN GERIATRICS SOCIETY, 2012).

O presente estudo tem como objetivo analisar a presença de prescrição de MPIs por meio dos critérios de Beers de
2015 em um grupo de idosos participantes de um programa de gerenciamento de doenças crônicas (GDC) de um plano de saúde na cidade de São Paulo, SP, Brasil.

\section{Material e métodos}

Trata-se de um estudo exploratório e descritivo, realizado no período de setembro a dezembro de 2015. A amostra foi constituída por idosos a partir de 60 anos, não institucionalizados, vinculados a um plano de saúde na cidade de São Paulo e participantes de um programa de GDC.

O programa de GDC é elaborado com os objetivos de promover a saúde e prevenir doenças. Para isso, busca aumentar o envolvimento do idoso com sua(s) enfermidade(s), estimulando o autocuidado e prevenindo crises agudas e complicações das DCNTs, esperando, assim, interferir de maneira benéfica no curso natural da doença. Todos os participantes do programa devem ter pelo menos uma DCNT diagnosticada, devem estar em acompanhamento médico para sua enfermidade e em tratamento (MANSO; BIFFI; GERARDI, 2015).

Para esta pesquisa, os idosos participantes foram inicialmente contatados, tomando ciência da voluntariedade, do ingresso opcional e do sigilo dos seus dados. Somente após todos assinarem o termo de consentimento livre e esclarecido, uma equipe de enfermeiros treinados coletou os dados nas residências de cada participante durante a realização das atividades do programa. As seguintes variáveis foram consideradas: idade, sexo, número e tipo de fármacos prescritos 
por idoso, categoria terapêutica destes e presença de prescrição medicamentosa inadequada.

Foram excluídos todos os indivíduos que apresentavam informações incompletas e prescrições de produtos como: creme hidratante, colírio, protetor solar, vitaminas, fitoterápicos e medicamentos em fórmulas de manipulação.

Para classificar os MPIs, foi utilizada a classificação da American Geriatrics Society - Critério de Beers, versão de 2015. Nessa classificação, os medicamentos são separados de acordo com a sua classe terapêutica e o seu princípio ativo, independentemente do diagnóstico e da condição clínica (AMERICAN GERIATRICS SOCIETY, 2015). A análise comparativa com os critérios de Beers foi realizada por meio do programa Microsoft Excel, com a ferramenta Tabela Dinâmica.

O projeto de pesquisa foi aprovado pela Comissão de Ética em Pesquisa do Centro Universitário São Camilo sob o parecer $\mathrm{n}^{\circ}{ }^{\mathrm{0}}$ 1.251.656.

\section{Resultados}

Foram analisadas as prescrições realizadas a 1.428 idosos participantes do programa de GDC e vinculados a um plano de saúde. O grupo pesquisado foi composto, em sua maioria, por mulheres $(\mathrm{n}=936,65,6 \%)$, conforme visualiza-se na Tabela 1, que apresenta os dados demográficos dos participantes da pesquisa. A idade média encontrada para o grupo foi de 78,5 anos, sendo que as mulheres participantes do grupo são mais longevas do que os homens.
Tabela 1 - Características sociodemográficas de um grupo de idosos pertencentes ao programa de GDC, São Paulo, SP, 2015

\begin{tabular}{l|l}
\hline \multicolumn{1}{c|}{ Variáveis } & \multicolumn{1}{c}{ Valores } \\
\hline $\begin{array}{l}\text { Idade média } \\
\text { Sexo feminino }\end{array}$ & $78,5 \pm 8,08[60-101]$ \\
$65,6 \%(936)$ \\
$\begin{array}{l}\text { Idade média do sexo } \\
\text { feminino }\end{array}$ & $76,4 \pm 8,3[60-101]$ \\
$\begin{array}{l}\text { Idade média do sexo } \\
\text { masculino }\end{array}$ & $74,7 \pm 7,4[60-97]$ \\
\hline
\end{tabular}

Fonte: elaboração dos autores.

Os resultados são apresentados como \%(n) para as variáveis categóricas e média $\pm \mathrm{DP}$ [mínima-máxima] para as variáveis contínuas.

Obteve-se um total de 5.995 fármacos prescritos por médicos, com média de medicamentos utilizados por idoso de 4,2, variando de 1 a 13 fármacos/ receituário. Dessas prescrições, 1.220 $(20,3 \%)$ foram consideradas como MPIs.

Os MPIs foram agrupados e analisados, encontrando-se $481(39,4 \%)$ inibidores de bomba de prótons (IBPs); $162(13,3 \%)$ medicamentos antidiabéticos; 132 (10,8\%) benzodiazepínicos (BZPs) de ação curta, intermediária e longa; 127 (10,3\%) anti-inflamatórios não hormonais (AINEs); 83 (6,8\%) anti-histamínicos de primeira geração; e 67 $(5,4 \%)$ relaxantes musculoesqueléticos, como apresentado na Tabela 2 , em que se observam a categoria terapêutica e o tipo de medicamento prescrito. Os demais fármacos, surgiram em frequências inferiores a $1 \%$ e, por serem múltiplos, não foram incluídos na tabela.

Os IBPs, muito utilizados para tratar disfunções gastrointestinais, 
tiveram $481(39,4 \%)$ prescrições, sendo caracterizados como o medicamento mais prescrito dentre todos. Na categoria dos fármacos endocrinológicos, a insulina móvel teve $162(13,3 \%)$ prescrições, tendo a segunda maior frequência de prescrições. Ainda nessa categoria, o estrógeno com ou sem progesterona teve $16(1,3 \%)$ prescrições.
O ácido acetilsalicílico (AAS) foi encontrado em $94(7,7 \%)$ prescrições, no entanto, em doses relacionadas à sua ação trombolítica (<325 mg/dia), incluídas na categoria terapêutica de dor. Os relaxantes musculoesqueléticos apresentaram $67(5,4 \%)$ prescrições, sendo o fármaco mais prescrito o carisoprodol, com $32(2,6)$ receitas.

Tabela 2 - Distribuição dos MPIs encontrados em um grupo de idosos vinculados a um plano de saúde, segundo critérios de Beers de 2015, São Paulo, SP, 2015

\begin{tabular}{|c|c|c|c|}
\hline & Categoria terapêutica & Medicamento & $\begin{array}{l}\%(n) \text { de } \\
\text { prescrições }\end{array}$ \\
\hline \multirow[t]{2}{*}{ Anticolinérgico } & \multirow[t]{2}{*}{ Anti-histamínico de primeira geração } & Dimenidrinato & $1,1 \%(13)$ \\
\hline & & Prometazina & $4,3 \%(53)$ \\
\hline Anti-infeccioso & Antibiótico & Nitrofurantoína & $1,4 \%(17)$ \\
\hline Cardiovascular & Bloqueador alfa 1 periférico & Doxazosina & $3,0 \%(37)$ \\
\hline \multirow[t]{5}{*}{ Ação central } & $\begin{array}{l}\text { Inibidor seletivo da recaptação de sero- } \\
\text { tonina (ISRS) }\end{array}$ & Paroxetina & $1,2 \%(15)$ \\
\hline & \multirow[t]{3}{*}{$\begin{array}{l}\text { Benzodiazepínico (de ação curta, inter- } \\
\text { mediária, longa) }\end{array}$} & Alprazolam & $2,4 \%(29)$ \\
\hline & & Lorazepam & $1,2 \%(15)$ \\
\hline & & Clonazepam & $6,6 \%(80)$ \\
\hline & Hipnóticos não benzodiazepínicos & Zolpidem & $2,5 \%(31)$ \\
\hline \multirow[t]{2}{*}{ Endócrino } & \multirow[t]{2}{*}{ Antidiabético } & Insulina (escala móvel) & $13,3 \%(162)$ \\
\hline & & $\begin{array}{l}\text { Estrógeno com ou sem } \\
\text { progestágenos }\end{array}$ & $1,3 \%(16)$ \\
\hline Gastrointestinal & Inibidor da bomba de prótons (IBP) & Omeprazol & $39,4 \%(481)$ \\
\hline \multirow[t]{5}{*}{ Dor } & \multirow[t]{2}{*}{$\begin{array}{l}\text { Anti-inflamatórios não hormonais (AINEs) } \\
\text { orais não seletivos para inibição da COX }\end{array}$} & Aspirina $>325 \mathrm{mg} / \mathrm{dia}$ & $7,7 \%(94)$ \\
\hline & & Meloxicam & $1,1 \%(14)$ \\
\hline & \multirow[t]{3}{*}{ Relaxantes musculoesqueléticos } & Carisoprodol & $2,6 \%(32)$ \\
\hline & & Ciclobenzaprina & $1,2 \%(15)$ \\
\hline & & Orfenadrina & $1,6 \%(20)$ \\
\hline
\end{tabular}

Fonte: elaboração dos autores. 
Quanto aos medicamentos de ação central, os BZPs de curta, intermediária e longa ação tiveram $132(10,8 \%)$ ocorrências, com um predomínio de 80 $(6,6 \%)$ prescrições aos de longa ação, como clonazepam. Os hipnóticos não BZPs representaram $31(2,5 \%)$ receitas. Dos anticolinérgicos, $83(6,8 \%)$ foram anti-histamínicos de primeira geração, sendo prometazina $(4,3 \%)$ e dimenidrinato $(1,1 \%)$ os mais receitados. Em relação aos medicamentos de categoria cardiovascular, o bloqueador alfa 1 (doxazosina) teve 37 (3\%) prescrições. Em relação aos anti-infecciosos, a nitrofurantoína $(1,4 \%)$ foi a única medicação prescrita para o grupo de idosos participantes da pesquisa.

\section{Discussão}

Os critérios de Beers foram criados com base em medicamentos americanos, e somente $60 \%$ dos medicamentos citados são comercializados no Brasil, mas, apesar disso, ainda são os critérios preferencialmente utilizados (LOPES et al., 2016).

Observou-se uma elevada prevalência de MPIs neste estudo, o que está de acordo com o índice encontrado em outros estudos realizados com idosos no segmento do sistema de saúde brasileiro $(33,4 \%)$ (MANSO; BIFFI; GERARDI, 2015; MUNIS et al., 2017). Já, para usuários idosos atendidos pelo sistema público, a literatura refere uma grande variabilidade de prescrição de MPIs, chegando a atingir $62,8 \%$ do total de medicamentos (MUNICK; ARAUJO, 2012).
Os MPIs mais prescritos aos idosos participantes deste estudo foram os relacionados à categoria gastrointestinal $\mathrm{e}$, dentre eles, os IBPs. Esses medicamentos têm como efeitos colaterais cefaleia, diarreia, tontura, sonolência, confusão mental, impotência, ginecomastia e dor muscular e articular. Em longo prazo, os IBPs podem causar efeitos indesejados, principalmente na população idosa, e estão relacionados ao risco de osteoporose/fraturas, demências, infecção por Clostridium difficile e atrofia gástrica, recentemente, também têm sido associados ao desenvolvimento de câncer gástrico, mesmo em casos com erradicação bem-sucedida de Helicobacter pylori (AMERICAN GERIATRICS SOCIETY, 2015; CHEUNG et al., 2017).

Os IBPs reduzem a absorção de alguns minerais e vitaminas, entre eles cálcio e vitamina B12, ao modificarem o $\mathrm{pH}$ do estômago. A redução de cálcio predispõe a osteopenia, osteoporose e, consequentemente, fraturas (CHINZON; CHINZON; RUZZI, 2015; OLIVEIRA et al., 2016). Já a deficiência de vitamina B12 promove danos no sistema nervoso central e periférico, ocasionando um quadro de demência (CHINZON; CHINZON; RUZZI, 2015). Ao aumentar o pH do estômago, esses medicamentos favorecem a proliferação da bactéria Clostridium difficile, o que pode levar a um quadro grave de diarreia (SILVA JUNIOR, 2012). Nos idosos, essa infecção torna-se mais grave, pois predispõe à desidratação.

Os critérios de Beers de 2015 recomendam que os IBPs sejam utilizados 
por no máximo oito semanas. Porém, em situações como no caso de pacientes que fazem uso contínuo de corticoides ou AINEs, com esôfago de Barret, esofagite erosiva ou quando houve falha na tentativa de suspender o IBP ou trocá-lo por bloqueador $\mathrm{H}_{2}$, não é possível suspender a medicação (AMERICAN GERIATRICS SOCIETY, 2015).

Os IBPs são fármacos que, muitas vezes, são prescritos para combater efeitos colaterais de outros fármacos, ou seja, ocorre um ciclo vicioso denominado cascata iatrogênica, em que, a cada sintoma que surge decorrente de um efeito colateral não identificado, emprega-se um novo medicamento (MUNICK; ARAUJO, 2012).

É muito alto o consumo de psicotrópicos no Brasil, em especial o de BZPs, principalmente em pacientes em uso de polifarmácia e em mulheres (MANSO; OLIVEIRA; BIFFI, 2016). Os BZPs são indicados para manejo de distúrbios psiquiátricos, como relaxantes musculares e anticonvulsivantes, porém devem ser usados por curto tempo, não ultrapassando três meses (MANSO; BIFFI; GERARDI, 2015; AMERICAN GERIATRICS SOCIETY, 2015; LOPES et al., 2016).

Os BZPs têm composição lipossolúvel, o que permite uma rápida absorção e distribuição para o sistema nervoso central (SNC) e uma diminuição da metabolização hepática, sendo que os idosos possuem uma maior sensibilidade a esses efeitos. Esses medicamentos podem ocasionar sintomas como sonolência diurna, diminuição da coordenação motora, alteração da memória, tontura, zumbidos, delirium, quedas e fraturas, reação paradoxal, intoxicação, além do risco de dependência. $\mathrm{O}$ uso crônico em idosos parece aumentar o risco de demência, principalmente da doença de Alzheimer (MANSO; BIFFI; GERARDI, 2015; AMERICAN GERIATRICS SOCIETY, 2015; LOPES et al., 2016).

A ação depressora no SNC dos BZPs, quando associados com outros fármacos, como barbitúricos e anti-histamínicos, pode ter seu efeito sedativo potencializado e até inibir o centro respiratório bulbar, induzindo a uma insuficiência respiratória (VIEL et al., 2014).

Os anti-histamínicos de primeira geração possuem ação anticolinérgica, antidopaminérgica e antisserotoninérgica. Eles são as medicações mais utilizadas no tratamento de reações alérgicas graves e possuem grande efeito neuropsicológico. Seus efeitos são sonolência, sedação, fadiga, boca seca, constipação, toxicidade, redução das funções cognitivas, confusão mental, diminuição da memória e do desempenho psicomotor, o que se associa ao risco de quedas em idosos (MANSO; BIFFI; GERARDI, 2015; AMERICAN GERIATRICS SOCIETY, 2015).

Como os BZPs e os anti-histamínicos têm ação no sistema nervoso central, quando associados, eles podem comprometer as atividades motoras, causando tontura, zumbido, cansaço, fadiga, visão embaçada, tremores e, consequentemente, quedas. Esses medicamentos devem ser evitados em idosos, principalmente devido ao risco de quedas (AMERICAN 
GERIATRICS SOCIETY, 2015; VIEL et al., 2014).

A queda, que pode estar associada a fraturas, leva o idoso à incapacidade funcional, o que o torna dependente em muitas de suas atividades de vida diária, podendo culminar com as síndromes da fragilidade e da imobilidade. Como consequência, há um grande impacto sobre a dependência e a autonomia do idoso (MANSO; BIFFI, 2014).

Nesta pesquisa, 10,3\% dos MPIs eram da classe dos AINEs. Esses medicamentos são muito utilizados por idosos, principalmente a aspirina, utilizada como antiagregante plaquetário (MANSO; BIFFI; GERARDI, 2015; AMERICAN GERIATRICS SOCIETY, 2015; OLIVEIRA et al., 2016; MANSO; BIFFI, 2014). Os AINEs podem alterar a pressão arterial e descompensar doenças cardiovasculares e renais preexistentes, pois reduzem o fluxo sanguíneo renal, a taxa de filtração glomerular, inibem a ação do hormônio antidiurético e a reabsorção de íons cloreto (MANSO; BIFFI; GERARDI, 2015). A prescrição de AINEs deve ser criteriosa, pois a hipertensão arterial e as doenças cardiovasculares são muito prevalentes em idosos.

O uso prolongado de anti-inflamatórios pode levar a lesões gástricas, que, na maioria das vezes, são tratadas com IBPs, ou seja, o efeito indesejado do primeiro fármaco é combatido com adição de outro medicamento também inapropriado para idosos, como já mencionado (SILVA; MENDONÇA; PARTATA, 2014).

Outra classe de MPI com prevalência relevante neste estudo é a dos relaxantes musculares. Eles possuem como efeitos colaterais risco elevado de fraturas em idosos e sonolência, por isso sua inclusão nos critérios de Beers (AMERICAN GERIATRICS SOCIETY, 2012).

A insulina é amplamente utilizada pela população, principalmente por idosos. Segundo os critérios de Beers, o uso de insulina em idosos baseado nos níveis de glicemia deve ser evitado, pois aumenta o risco de hipoglicemia e, dessa forma, deve ser cautelosamente administrada, portanto, cabe ao médico avaliar os riscos e os benefícios (AMERICAN GERIATRICS SOCIETY, 2012, 2015).

Neste estudo, observou-se um índice de MPIs preocupante, já que se trata de um grupo de idosos portadores de, pelo menos, uma DCNT e com faixa etária elevada, o que evidencia uma maior vulnerabilidade a institucionalizações, RAMs, hospitalizações e morte.

Este estudo apresentou algumas limitações, já que foi realizado em um grupo de idosos assistidos por um plano de saúde e acompanhados por um programa de GDC, o que não permite a generalização dos resultados. Destaca-se, ainda, que não foi considerada a presença de automedicação, RAMs e hospitalizações.

\section{Conclusão}

O presente estudo observou um alto índice de MPIs e de medicamentos em uso contínuo em um grupo de idosos vinculados a um plano de saúde do município de São Paulo. Os pesquisadores esperavam encontrar índices menores 
de MPIs, já que se tratavam de idosos acompanhados simultaneamente por médicos e por um programa de GDC.

O grupo investigado encontrava-se, por suas próprias características clínicas, uso de MPIs e polifarmácia, em situação de vulnerabilidade biológica, com predisposição a desfechos relacionados a RAMs, como perda de capacidade funcional, confusão mental, dor muscular e articular, impotência, ginecomastia, cefaleia, diarreia, sonolência, tontura, zumbidos, delirium, intoxicação, reação paradoxal, diminuição da coordenação motora, osteoporose, quedas e fraturas, comprometendo a qualidade de vida destas pessoas.

\section{Use of beers criteria for the evacuation of prescriptions in older adults with chronic diseases linked to a health plan}

\section{Abstract}

Senescence is a natural process that alters pharmacokinetic and pharmacodynamics of medications, it is particularly important when it comes to an elderly country like Brazil. Associated with age there is an increase in cronical non-transmissible diseases and consequently the use of various medications. The polypharmacy increases the risk of adverse reactions, especially when associated with the prescription of potentially inappropriate medications (MPI) for elderly. The present study aims to evaluate the prescription of MPI through the 2015 Beers criteria in a group of elderly with cronical diseases linked to a health insurance in São Paulo, Brazil. It is an exploratory, descriptive study conducted through the analysis of 5,995 medical prescriptions, during September and December, 2015. The medications were evalua- ted by the Beers criteria reviewed in 2015 . It was observed that of the 5,995 medical prescriptions to 1,428 elderly, $20.3 \%$ were considered MIP. The most prescribed drugs were $39.4 \%$ (481) proton pump inhibitor (PPI), 13.3\% (162) of antidiabetic, 10.8\% (132) benzodiazepines (BZP), intermediate and long-acting, $10.3 \%$ (127) for nonsteroidal anti-inflammatory drugs (NSAIDs) and $6.8 \%$ (83) were of the first-generation antihistamine class. The group studied presented a high PMPI index and of medications in continuous use by elderly. It is a population group in risk, because of their clinical particularities predisposing to unfavorable biophycosocial outcomes.

Keywords: Drug prescriptions. Potentially Inappropriate medication list. Assistance to elderly health. Health insurance.

\section{Referências}

AMERICAN GERIATRICS SOCIETY. American Geriatrics Society 2015 Updated Beers Criteria for Potentially Inappropriate Medication Use in Older Adults. Journal of the American Geriatrics Society, New York, v. 63 , n. 11, p. 2227-2246, Nov. 2015.

American Geriatrics Society Updated Beers Criteria for Potentially Inappropriate Medication Use in Older Adults. Journal of the American Geriatrics Society, New York, v. 60 , n. 4, p. 616-631, Apr. 2012.

CARVALHO, M. F. C. et al. Polifarmácia entre idosos do município de São Paulo Estudo SABE. Revista Brasileira de Epidemiologia, São Paulo, v. 15, n. 4, p. 817-827, jan. 2012.

CHAIMOWICZ, F. et al. Saúde do idoso. 2. ed. Minas Gerais: NESCON UFMG, 2013.

CHEUNG, K. S. et al. Long-term próton pump inhibitors and risk of gastric cancer development after treatment for Helicobacter pylori: a population-based study. Gut, London, v. 67, n. 1, p. 28-35, Jan. 2017. 
CHINZON, D.; CHINZON, M.; RUZZI, A. M.; A prática clínica em doenças reumáticas: perfil de segurança da terapia com inibidores de bomba de prótons (IBP). Clínica e Terapêutica Gastroenterologia, São Paulo, v. 1, n. 1, p. 11-16, maio 2015.

CONSTANTINO, J.; FIGUEIREDO, I. V. Polimedicação no idoso. Relato de um caso: Angina de peito e comorbilidades. Acta Farmacêutica Portuguesa, Coimbra, v. 4, n.1, p. 3-16, jan./jun. 2015.

FECHINE, B. R. A.; TROMPIERI N. O processo de envelhecimento: as principais alterações que acontecem com o idoso com o passar dos anos. Inter Science Place, Ceará, v. 1 , n. 7, p. 106-194, mar. 2012.

GLASSOCK, R.; DENIC, A.; RULE A. D. Quando os rins envelhecem: um ensaio em nefro-geriatria. Jornal Brasileiro de Nefrologia, São Paulo, v. 39, n. 1, p. 59-64, mar. 2017.

GORZONI, M. L.; FABBRI, R. M. A.; PIRES, S. L. Medicamentos potencialmente inapropriados para idosos. Revista da Associação Médica Brasileira, São Paulo, v. 58, n. 4, p. 442-446, ago. 2012.

LOPES, L. M. et al. Utilização de medicamentos potencialmente inapropriados por idosos em domicílio. Ciência \& Saúde Coletiva, Belo Horizonte, v. 21, n. 11, p. 3429-3438, nov. 2016.

MANSO, M. E. G.; BIFFI, E. C. A. Manual da Liga de Estudos do Processo de Envelhecimento. São Paulo: Martinari, 2014.

MANSO, M. E. G.; BIFFI, E. C. A.; GERARDI, T. J. Prescrição inadequada de medicamentos a idosos portadores de doenças crônicas em um plano de saúde no município de São Paulo, Brasil. Revista Brasileira de Geriatria e Gerontologia, Rio de Janeiro, v. 18, n. 1, p. 151-164, mar. 2015.

MANSO, M. E. G.; GALERA, P. B. Perfil de um grupo de idosos participantes de um programa de prevenção de doenças crônicas. Estudos Interdisciplinares sobre o Envelhecimento, Porto Alegre, v. 20, n. 1, p. 57-71, abr. 2015.
MANSO, M. E. G.; OLIVEIRA, H. S. B.; BIFFI, E. C. A. Gênero e polifarmácia: impacto em um grupo de idosos vinculados a um plano de saúde. In: CONGRESSO BRASILEIRO DE GERIATRIA E GERONTOLOGIA, 20. Resumos... Fortaleza, CE, 2016. p. 1233.

MUNICK, A. K. R.; ARAUJO, A. L. A. Avaliação dos medicamentos inapropriados prescritos para pacientes idosos em um hospital universitário. HU Revista, Juiz de Fora, v. 38, n. 3-4, p. 231-240, jul./dez. 2012.

MUNIS, E. C. S. et al. Analysis of medication use by elderly persons with supplemental health insurance plans. Revista Brasileira de Geriatria e Gerontologia, Rio de Janeiro, v. 20, n. 3, p. 375-387, maio/jun. 2017.

OLIVEIRA, M. G. et al. Consenso brasileiro de medicamentos potencialmente inapropriados para idosos. Geriatrics, Gerontology and Aging, Rio de Janeiro, v. 10, n. 4, p. 168-181, out/dez. 2016.

SILVA, J. M.; MENDONÇA P. P.; PARTATA, A. K. Anti-inflamatórios não-esteroidais e suas propriedades gerais. Revista Científica do ITPAC, Araguaína, v. 7, n. 4, Pub. 5, out. 2014.

SILVA JUNIOR, M. Recentes mudanças da infecção por Clostridium difficile. Einstein, São Paulo, v. 10, n. 1, p. 105-109, mar. 2012.

VIEL, A. M. et al. Interações medicamentosas potenciais com benzodiazepínicos em prescrições médicas de pacientes hospitalizados. Revista de Ciências Farmacêuticas Básica e Aplicada, Araraquara, v. 35, n. 4, p. 589-596, out./dez. 2014. 